\title{
Community social valuation: use of nominal group technique in ranking of health conditions from two communities in Temeke and Moshi Districts in Tanzania
}

\author{
E. MAKUNDI ${ }^{1}$, O. NORHEIM ${ }^{2}$, L. HARAM ${ }^{2}$, P. SETEL $^{3}$ \& G. KVÅLE ${ }^{2}$ \\ ${ }^{1}$ National Institute for Medical Research, Dar es Salaam, Tanzania \\ ${ }^{2}$ University of Bergen, Norway \\ ${ }^{3}$ Adult Morbidity and Mortality Project, Dar es Salaam, Tanzania
}

\begin{abstract}
This study used the nominal group technique to explore societal value preferences in the ranking of health conditions from two communities in Temeke and Moshi districts in Tanzania. The nominal group technique was applied to a community of lay people including patients and community leaders. In this study we found a relatively high stability of ranking values across sites and informant groups. The nominal group technique was easy for lay people to understand and less time consuming compared to other methods used in health state valuation. The findings indicate that the nominal group technique can be used in the valuation process with a population of lay people to obtain societal preferences as a basis for priority setting in health. This study was limited to using criteria as a guide in the voting exercise, which may have framed respondent's final voting judgement. Further studies are needed to assess informant's responses and test validity and reliability of this method with larger sample size in different sites and informant groups. In conclusion, the nominal group technique may be considered to obtain societal preferences to compliment the current burden of disease data for priority setting.
\end{abstract}

\section{Introduction}

Assessment of community values is necessary both for measurement of burden of disease and resource allocation in order to make informed choices for health policy priorities. The importance of measuring individual preferences is well recognised among health professionals, but using patients' or society's values in decision-making is far from common practice (Froberg and Kane, 1989a).

Recently there has been a growing concern in public health and health economics that health state valuation measures should reflect the perceptions of the lay public, including patients (Nord, 1992; Gill and Feistein, 1994; Leplege and Sonia, 1997). Some scholars argue that quality of life can be suitably measured only by determining the opinions of patients and thereby supplementing the instruments developed by 'experts' (Nord, 1992). Many valuation techniques consist of abstract thought exercises in which people assign weights to life years in different health states. Several valuation techniques are currently in use including the simple rating scale, visual analogue scale, magnitude estimation, standard gamble, time trade-off and person trade-off. These techniques have been compared in several studies and a considerable difference in results has been observed (Torrance, 1986; Froberg and Kane, 1989a; Nord, 1992).

One of the approaches to quantifying disease burden in human populations for priority setting is the use of Disability Adjusted Life Years (DALYs)
(Murray and Lopez, 1996). DALYs provide a framework for different professions in determining priorities in health care and evaluating efficacy of interventions since they employ a time-based measure that allows comparability across diseases, risk factors and between morbidity and mortality (Murray and Lopez, 1996; 1997). Just like Quality Adjusted Life Years (QALYs), DALYs combine information about quality and quantity of life into a single number. The QALY approach uses weights on a scale going from 0 to 1 , with 'zero' being for "death" and 'one' for "full health" (Nord, 1999). The purpose of health state valuation in the context of burden of disease research is to get additional information on non-fatal health outcomes on human populations (Murray and Lopez, 1996). In the DALY development the person tradeoff valuation method is used to generate disability weights by a panel of 'health experts' who are considered to be familiar with health outcomes with regard to relative ranking of health states in health care.

Several scholars have expressed their concerns regarding conceptual and methodological problems regarding the DALY approach (Anand and Hanson, 1997; Sayers and Fliedner, 1997; WHO, 1998; Arnesen and Nord, 1999). One of the concerns is a need for broader representation in the DALY valuation methodology; to get a more socially representative picture expressing a particular society's' preferences (WHO, 1998). Another issue in the DALY calculation is the fact that, just like in many other health, state 
valuation studies the person trade-off method was based on proxies where the subjects are asked to consider states that they have not experienced themselves (Nord, 1992).

Several studies have shown that different responses in rating health conditions are given by different types of respondents: the general public, health care providers, individuals living in particular health states or these individuals' family members (Froberg and Kane, 1989a,b; Nord, 1995; Gary and Devlieger, 1999). The general pattern is that, health care providers tend to rate health states as worse than the general public, who in turn rate them worse than those living in the particular health state. However, policy makers and health state valuation experts are faced with the following dilemmas regarding whose preferences should be used (Kind and Dolan, 1995). Should greatest weight be given to the valuation of those currently receiving health care as patients? Should greatest weight be given to the valuation of healthy people as potential patients and taxpayers? What about those most directly affected by a particular policy or intervention? Such questions may sound more political than scientific but a more inclusive process to incorporate the important parts is necessary depending on the specific objective of the valuation.

This study was motivated by these concerns about involving patients and lay people in the valuation exercise, which may act as a supplement to using health experts in health state valuation. We used the nominal group technique to find societal preferences in the valuation process. The subjects involved in this study included patients/caregivers, women's group representatives and community leaders. Our main objective was therefore, to study the usefulness of the nominal group technique in eliciting societal preferences of health states, and to assess if the technique would yield consistent ranking of health conditions between different groups from a community of lay people in Tanzania.

\section{Materials and methods}

\section{Study site}

The study was carried out in Mamba Ward, Moshi Rural District in northern Tanzania and Mtoni Street, Temeke District in eastern Tanzania. The study comprised community leaders, patients or caregivers and women group representatives living in the two communities. Mamba and Mtoni wards were purposively selected to represent a rural and an urban setting with a relatively high and low economic status, respectively. Mtoni is a semi-urban area with relatively low economic status. Mamba on the other hand, constitutes an area with relatively high economic status (TDHS, 1992/93). The study communities were selected through simple random sampling, with the names of all the villages in the selected sites written on small pieces of paper and then put in a basket. The pieces of paper were shuffled and one was randomly selected in each site.

Four key informants were interviewed, two from each site. The key informants were selected when investigators were developing rapport in the study communities. Selection of participants in this study was done through a discussion with two key informants and one of the investigators. In total there were four groups, two at each site. While two groups consisted of patients or their caregivers and women group representatives, the other two groups consisted of community leaders. The patients/caregivers were selected on a basis of seven health conditions. The final number of participants included in this study varied slightly by each group but essentially the quota of eight was achieved in each group.

A total of seven health conditions were used, namely: blindness, deafness, infertility (not simply a sequel) and diarrhoea (these are among 22 indicator conditions in the Global Burden of Disease Study using the person-trade-off method). In addition, malaria, accidents and intestinal worms were used (these were chosen from 10 leading causes of health problems by health experts in a priority setting workshop in Arusha, Tanzania in February 1999). The selection criteria of the seven conditions included those, which were common and easy for lay people to understand, both chronic and acute in nature. In addition, infertility was chosen as it was considered unique and given social stigma associated to its assessment. Seven conditions were chosen in the ranking because the use of additional conditions was considered to be too much of a burden on the respondent.

The nominal group technique was carried out in a group session that lasted on average 90 minutes. One discussion session was done under a tree in the village, another inside a classroom and the other two in the meeting room at the village office. Each group was presented with a brief overview of the exercise and its purpose. Respondents were asked about their understanding of the seven conditions. The investigators used pictures and examples to aid the description of the health states. This was done in an attempt to make all respondents think about the same health states when they were given their valuations. Kiswahili, the national language in Tanzania, was used in the group sessions because it was well understood in the study areas. Manila cards containing the 
conditions were given to each participant. On the manila cards, the conditions/pictures were arranged in circles. This procedure was thought necessary in order to reduce bias as compared to presentation in linear form. Thereafter, number tags marked 1 to 7 were given to each participant for the ranking exercise.

At first, each individual did simple ranking of health conditions from most to least severe by putting number tags 1 (most severe) to 7 (least severe) on top of each condition on the manila card. Results for each participant were noted. Discussion was not allowed at this stage. Secondly, individual results from the ranking exercise were displayed on a board followed by a discussion. Participants gave reasons for their ranking so as to obtain clarity and air out points of view but not to resolve differences in opinion.

A third step was a voting exercise. A tentative guide for social valuation of health states was explained to the group just before voting, including consideration of social stigma, effects to daily social life, magnitude and severity of the problem. The voting process was done through secret voting by using pieces of paper. Participants were asked to select what they considered the most severe condition in the list of seven through a self-elimination method. Starting with seven conditions participants voted six times. Prior to the voting exercise it was agreed that, when two or more health conditions had equal votes, the voting exercise would be repeated. Lastly the results were written on the board. Participants were asked to compare voting results with their individual ranking.

\section{Data analysis}

Data from discussion groups were collated and the seven health conditions were ranked in the order of priority from 1-7. Results from voting exercises were put in a table to facilitate group comparisons. The overall collective voting was obtained by adding the rank values of each condition from both sites, and sums were taken. Thereafter, the data from 32 individual ranks were entered into a spreadsheet. A sum of the ranks for each condition was taken. A ranking order from voting exercises and simple ranking was obtained by arranging the sums in ascending order. The condition scoring the least sum was considered as the most severe health condition, while the one scoring the largest sum was taken as the least severe health condition.

\section{Results}

Overall, the votes from four group sessions indicate a stable-voting pattern of health conditions in the two sites. There was a slight variability in the voting pattern for intermediate positions especially for accidents and intestinal worms than at both ends. Malaria was considered most severe by three out of four groups and second in one group. Blindness and infertility were considered least severe in all four groups. There were differences in the voting pattern between patients and community leaders in Mamba compared to that of Mtoni. For instance, whereas in Mamba diarrhoea was considered most severe by patients, the community leaders perceived malaria as the most severe. While community leaders considered blindness the least severe condition, patients considered infertility to be the least severe (Table 1).

Table 1: Collective ranking of health conditions by sites and discussion groups (sums of collective ranking in brackets)

\begin{tabular}{|c|c|c|c|c|c|}
\hline \multirow[t]{2}{*}{ Condition } & \multicolumn{2}{|c|}{ Patients/Caregivers } & \multicolumn{2}{|c|}{ Community leaders } & \multirow[t]{2}{*}{ Overall ranking } \\
\hline & Mtoni & Mamba & Mtoni & Mamba & \\
\hline Malaria & 1 & 2 & 1 & 1 & $1(5)$ \\
\hline Diarrhoea & 2 & 1 & 2 & 2 & $2(7)$ \\
\hline Accidents & 3 & 4 & 3 & 3 & $3(13)$ \\
\hline Intestinal worms & 4 & 3 & 4 & 4 & $4(15)$ \\
\hline Deafness & 5 & 5 & 5 & 5 & $5(20)$ \\
\hline Blindness & 6 & 6 & 6 & 7 & $6(25)$ \\
\hline Infertility & 7 & 7 & 7 & 6 & $7(27)$ \\
\hline $\mathrm{N}$ & 8 & 8 & 8 & 8 & 32 \\
\hline
\end{tabular}

*The least sum indicates highest priority and the largest indicates lowest priority. Numbers 1-7 indicate the order of priority. Collective ranking stands for results from voting by either groups or sites as shown in Table 2 below. 
The results of the voting exercise for patients, caregivers and women group representatives for Mtoni are shown in Table 2. Seven respondents voted malaria as the most severe condition in Mtoni while one person voted accidents as the most severe condition. Respondents had to vote twice for accidents and intestinal worms because in the initial voting, the two conditions received the same score.

Individual ranking showed more variability in the intermediate positions than at both ends (Table 3). Three discussion groups considered malaria and diarrhoea to be the most severe. Intestinal worms infestation was ranked second by community leaders in Mamba. All the four groups considered infertility least severe. There was a slight variation in the ranking pattern between community leaders and patients in Mamba compared to that of Mtoni. Whereas for example, diarrhoea was ranked second by patients in Mamba, the community leaders ranked it number three. In Mtoni, accidents were considered more severe by patients and ranked third. Accidents scored a fourth position among community leaders. In this area, intestinal worms and accidents tied-up by scoring the same values and they were ranked in the third position.
The overall ranking order based on the two methods was stable especially for malaria, blindness and infertility conditions in Mamba, and for almost all conditions in Mtoni (Table 4). There was a slight difference in the ranking order between the two methods in Mamba especially for the conditions ranked in the intermediate positions. Whereas malaria and diarrhoea were ranked first and second by patients and caregivers in Mamba in individual ranking, in the collective voting on the other hand, diarrhoea was ranked first and malaria second. Infertility, accidents and intestinal worms showed the same ranking pattern.

The voting and individual ranking patterns for community leaders in Mamba showed some variability as well. For example, whereas blindness was ranked fifth in the individual ranking, it was ranked seventh in the collective voting. However, there was a different pattern when collective and individual ranking orders were compared between the two groups at Mtoni. Collective and individual ranking showed a similar pattern in almost all positions for the two groups. Only accidents and intestinal worms, which were ranked in third position by patients and caregivers in the individual ranking, differed slightly in collective ranking where intestinal worm infestation was ranked fourth.

Table 2: Results of voting exercise in Mtoni and Temeke (Total number of votes in brackets)

\begin{tabular}{lll}
\hline Rank order & Health Condition & Number of votes \\
\hline 1 & Malaria & Malaria (7) versus accidents (1) \\
2 & Diarrhoea & Diarrhoea (4) versus accidents (2), intestinal worms (2) \\
3 & Accidents & Accidents (6) versusintestinal worms (2) \\
4 & Intestinal worms & Intestinal worms (6) versus infertility (1), blindness (1) \\
5 & Deafness & Deafness (4) versus blindness (3), infertility (1) \\
6 & Blindness & Blindness (5) versus infertility (3) \\
7 & Infertility & \\
\hline
\end{tabular}

*Key: Numbers 1-7 indicate the order of priority

Table 3: Individual ranking of health conditions by study site and discussion groups (sums of individual ranking in brackets)

\begin{tabular}{|c|c|c|c|c|c|}
\hline \multirow[t]{2}{*}{ Health condition } & \multirow[t]{2}{*}{ Overall ranking } & \multicolumn{2}{|c|}{ Patients/caregivers } & \multicolumn{2}{|c|}{ Community leaders } \\
\hline & & Mtoni & Mamba & Mtoni & Mamba \\
\hline Malaria & $1(47)$ & $1(10)$ & $1(13)$ & $1(13)$ & $1(11)$ \\
\hline Diarrhoea & $2(86)$ & $2(25)$ & $2(14)$ & $2(22)$ & $3(25)$ \\
\hline Intestinal worms & $3(109)$ & $3(33)$ & $3(22)$ & $4(33)$ & $2(21)$ \\
\hline Accidents & $4(128)$ & $3(33)$ & $4(35)$ & $3(29)$ & $4(31)$ \\
\hline Blindness & $5(164)$ & $6(40)$ & $5(45)$ & $6(42)$ & $5(37)$ \\
\hline Deafness & $6(165)$ & $5(39)$ & $6(47)$ & $5(35)$ & $6(44)$ \\
\hline Infertility & 7 (187) & $7(42)$ & $7(49)$ & $7(46)$ & $7(50)$ \\
\hline
\end{tabular}


When the overall individual ranking was compared to the overall collective voting between Mamba and Mtoni communities, there was some variability especially in the intermediate positions. Malaria, diarrhoea and infertility showed the same ranking pattern. Accidents and intestinal worms interchanged third and fourth positions while deafness and blindness interchanged fifth and sixth positions. for discussion and achieving a consensus through voting in the group. Though this study did not measure respondents' satisfaction, most of the respondents expressed positive feelings.

The nominal group technique was chosen because it was considered participatory and easy for lay participants to understand (McMurray, 1994). In addition, no technical medical knowledge was

Table 4: Comparison of the rank order between collective ranking technique and individual ranking in Mamba and Mtoni communities

\begin{tabular}{|c|c|c|c|c|c|c|c|c|c|c|}
\hline \multirow[t]{2}{*}{$\begin{array}{l}\text { Health } \\
\text { condition }\end{array}$} & \multirow{2}{*}{$\begin{array}{l}\text { Collective } \\
\text { ranking } \\
\text { order } \mathrm{N}= \\
32\end{array}$} & \multirow{2}{*}{$\begin{array}{l}\text { Individual } \\
\text { ranking } \\
\text { order } \\
\mathrm{N}=32\end{array}$} & \multicolumn{2}{|c|}{$\begin{array}{l}\text { Patient } \\
\text { caregiver in } \\
\text { Mamba,N=8 }\end{array}$} & \multicolumn{2}{|c|}{$\begin{array}{l}\text { Community } \\
\text { leaders in Mamba, } \\
\mathrm{N}=8\end{array}$} & \multicolumn{2}{|c|}{$\begin{array}{l}\text { Patient } \\
\text { caregiver in } \\
\text { Mtoni } N=8\end{array}$} & \multicolumn{2}{|c|}{$\begin{array}{l}\text { Community } \\
\text { leaders in mtoni }\end{array}$} \\
\hline & & & IR & CV & IR & $\mathrm{CV}$ & IR & CV & IR & CV \\
\hline Malaria & 1 & 1 & 1 & 2 & 1 & 1 & 1 & 1 & 1 & 1 \\
\hline Diarrhoea & 2 & 2 & 2 & 1 & 3 & 2 & 2 & 2 & 2 & 2 \\
\hline Accidents & 3 & 4 & 4 & 4 & 4 & 3 & 3 & 3 & 3 & 3 \\
\hline Worms & 4 & 3 & 3 & 3 & 2 & 4 & 3 & 4 & 4 & 4 \\
\hline Deafness & 5 & 6 & 6 & 5 & 6 & 5 & 5 & 5 & 5 & 5 \\
\hline Blindness & 6 & 5 & 5 & 6 & 5 & 7 & 6 & 6 & 6 & 6 \\
\hline Infertility & 7 & 7 & 7 & 7 & 7 & 6 & 7 & 7 & 7 & 7 \\
\hline
\end{tabular}

Key: IR = individual ranking; $\mathrm{CV}=$ collective voting

\section{Discussion}

This study has sought to explore the application of the qualitative nominal group technique on a sample of patients/caregivers and community leaders. The nominal group technique appears to be a useful methodological approach to elicit societal value preferences of health conditions in a community of lay people. The technique was easy for lay people to understand and less time consuming relative to other valuation techniques.

The strength of the nominal group technique used in this study is the interactive nature of the group sessions and the increased freedom of judgement as indicated through voting. The discussion sessions were quite interactive and participants were free and independent to discuss their views. The most interesting stage was the voting exercise where the participants were highly excited. The secret voting process was democratic, which seemed to enhance freedom of expression of ideas. The group sessions were done on the basis of deliberation, where a consensus is achieved on the basis of discussion. In addition, the fact that the outcomes of all procedures were clearly documented and understood by all members emphasised the practicality of the procedure. The technique therefore offers a unique opportunity required. In this technique, the number of conditions was limited to seven based on the assumption that it is difficult for humans to process more than nine pieces of information simultaneously (Miller, 1956; Froberg and Kane, 1989a; Nord, 1992).

Another point to consider in this study is that, the nominal group technique explicitly requires each member of the group to have an equal input in both the initial individual ranking and the voting stages. People feel more committed to a group if they have a chance to influence its thinking (Walton, 1985). It is important to note that, while focus group discussions may allow some individual expression, some individuals may be more reluctant to participate than others. This may lead to some participants to dominate the discussion. In addition, the technique gives an opportunity for participants and investigators in the same session to undertake two exercises at the same time, simple ranking and voting, and thereafter comparing the results. In other studies, such as those using survey techniques, while the informants provide vital information, sometimes they may not have the opportunity to see and compare results.

The nominal group technique when compared to other valuation techniques was relatively easy for a community of semi-literate respondents to understand. One study involving semi-literate women in 
quantifying the burden of illness and disease based on local perceptions was done in Cambodia. The Cambodia study combined the simple rating scale, visual analogue scales, person-trade-off method and standard gamble in the valuation exercise and showed the difficulty of involving lay people in these processes (WHO, 1998). It is not surprising that in the Cambodia study, no one completed a standard gamble valuation. Most individuals were reluctant to conduct any variant of the person-trade-off approach. All women completed simple ranking and visual analogue scale valuations. Given the level of abstraction required to use other scaling valuation methods such as persontrade-off and standard gamble, the nominal group technique is recommended in a population of lay people.

Our study has shown a stable ranking of societal values across groups and sites as indicated in voting results. The stability of ranking shows a relatively high level of agreement by different informants in both sites using the nominal group measurement method. The valuation seems to be based on prevalence, where informants gave more weight to the most prevalent common conditions such as malaria and diarrhoea in their community compared to rare conditions such as blindness and infertility. This was also confirmed during the discussion session between the individual ranking and the voting exercise when most participants viewed malaria and diarrhoea as the more life threatening diseases in their area. One possible explanation for the stability of ranking values is a tentative criterion, which was used as a guide prior to the voting stage which included consideration of social stigma, effects to daily social life, magnitude and severity of the problem. It is interesting to note that, the ranking pattern using simple ranking valuation method is also stable and is not very different from voting though a tentative criterion as a guide was not used.

It is worth noting that participants were reluctant to compare infertility with malaria saying it is a taboo to compare a condition caused by poverty to that resulting from God's will. This implies that individuals' preferences of health states are influenced by other concerns than those embedded within a biological understanding of disability. These accounts are discussed in detail elsewhere (Makundi et al., 2004).

Our findings indicate that the collective and individual ranking results of health conditions are stable across sites and informant groups, despite a slight variation especially in the intermediate positions. The ranking pattern between individual ranking and collective voting showed a slight variability in Mamba than in Mtoni. The design of this study was not set out to test for variations across groups, but this variability may be explained partly by the characteristics of the two-study populations. Mamba constituted a homogenous rural population with relatively high economic status as reflected through observation of modern houses (with corrugated iron sheet roofs), availability and accessibility of clean tap water, and presence of coffee and banana as permanent cash and food crops. Generally, more people were educated in Mamba than in Mtoni (data not shown). After the initial individual ranking and subsequent discussion, Mamba informants changed their ranking in the final voting. On the other hand, Mtoni constituted a heterogeneous urban population with relatively low incomes, as reflected in the people's lack of permanent incomes and poorly developed infrastructures. The discussion, which followed the initial individual ranking, did not seem to influence the final voting judgement in Mtoni as compared to Mamba.

The use of the nominal group technique in identifying problems and setting priorities in health care has been appreciated (McMurray, 1994; Maclachlan, 1996; Redman et al., 1997). The stability of results in the nominal group technique in this study has been shown in other studies using various methods in identifying health priorities and in valuation exercises. Redman et al. (1997) used the nominal group technique in identifying priorities for the National Breast Cancer Centre in Australia and found a reasonably high degree of agreement about a number of priorities across groups. Using the simple ranking method, Ustun et al. (1999), found a high stability in the rankings of 17 health conditions for $241 \mathrm{key}$ informants from 14 countries. In our study, using simple ranking and voting exercises in the nominal group technique we also found a consistent ranking of health conditions between different groups from a community of lay people in Tanzania.

Health state valuations have been shown to differ according to the experience of illness. A general rating pattern, according to Froberg and Kane (1989a) and Nord (1992) is that health care providers tend to rate health states worse than the general public, and the latter in turn, rate them worse than those living with a 
particular health condition. However, one important challenge that arises here is whose preferences should be used. For example, if the values of healthy members of public are taken, the severity weights may be overestimated. Likewise, if the values of the affected people, who have already coped with their situations, are taken, less importance may be attached to the degree of severity. Clearly, these are political rather than scientific issues but empirical evidence available highlights on the implications of choices made (Kind and Dolan, 1995). However, the current evidence points out that no systematic preference differences are found among rating groups due to demographic characteristics, but rather age and experience of health state being rated, seem to influence health state measurement (Torrance et al., 1982; Froberg and Kane, 1989a).

One limitation in this kind of valuation relates to the way the respondents were asked the questions. This may have caused framing effects in the valuation exercise. As noted earlier, the nominal group technique consisted of four steps namely simple ranking, discussion, voting and comparison of results. In the voting stage, a tentative criterion was used as a guide including consideration of social stigma, effects to daily social life, magnitude and severity of the problem. Magnitude of the health problem seems to have affected the voting more than severity of problem, social stigma and effects on daily social life. This may have limited respondent's independent judgement in the final voting exercise. On the other hand, the use of criteria in the voting exercise has an advantage to make respondents be familiar with health conditions in the same way during the voting process. The study indicates that conditions with a high prevalence in the community are given more weights than the more rare conditions. Thus, the way the method was used did not distinguish clearly between the severity and burden of the health condition. Although the ranking provides a rough indication of the burden of disease in the communities it does not provide a ranking of people's perception of severity as observed in our study.

Another limitation related to this method is the discussion session during the period between individual ranking and the voting exercise, which may have influenced the final voting judgement. On the other hand, the discussion session might be an advantage in the groups' deliberative process by reaching a consensus on the basis of discussion. This may be closer to the person trade-off-method used in the burden of disease study by Murray and Lopez (1996). Another limitation relates to the use of caregivers, which may not adequately represent patients' views in the valuations. When caregivers stand as proxies for patients, their views could be more representative of able-bodied people than patients they represent. Moreover, respondents felt alienated because the researchers introduced health conditions for the ranking exercise, which they considered not relevant as compared to other health problems in their area.

In conclusion, this exploratory study has outlined the application of the nominal group technique in assessing societal preferences in the ranking of health conditions. This method may be considered to advance societal preferences in the measurement of burden of disease in order to compliment the current burden of disease data used for priority setting in health care systems. However, the nominal group technique should 'not stand alone' as a research tool. This is because although the nominal group technique may add qualitative richness to quantitative methods, it may be inappropriate for generalisation as it only gives indepth information for a small number of people. Moreover, the use of criterion as a guide in the valuation process provides a basis for framing of respondents' judgement, which may limit their final decision making in the voting process. It is important to study how the ranking pattern would behave if the 
valuation uses more specific criteria or without using criteria, when a larger sample size is used from different sites and informant groups. It is therefore, necessary to explore further the application of the nominal group technique to generate disability weights from a community of lay people.

\section{Acknowledgements}

We would like to acknowledge Ms. Vivian Makule and Mr. Kazaura for excellent data collection and analysis, respectively. We are indebted to the key informants in Moshi and Temeke districts for their valuable information. Ms. Trude Arnesen, of the National Institute of Public Health at the University of Oslo and Professor Kristian Heggenhougen from the International Health Department at the Boston University are thanked for their comments on an earlier version of the manuscript. This study received financial assistance from the Norwegian Agency for Development Co-operation.

\section{References}

Anand, S. \& Hanson, K. (1997) Disability-adjusted life years: a critical review. Journal of Health Economics 16, 685-702.

Arnesen, T. \& Nord, E. (1999) The value of DALY life: problems with ethics and validity of disability adjusted life years. British Medical Journal 319, 1423-1425.

Froberg, D.G. \& Kane, R.L. (1989a) Methodology for measuring health-state preferences- I: Measurement strategies. Journal of Clinical Epidemiology 42, 345-354.

Froberg, D.G. \& Kane, R.L. (1989b) Methodology for measuring health-state preferences.- II: Scaling methods. Journal of Clinical Epidemiology 42, 459-471.
Gary, A.L. \& Devlieger, P.J. (1999) The disability paradox: high quality of life against all odds. Social Science and Medicine 48, 977-988.

Gill, T.M. \& Feistein, A.R. (1994) A critical appraisal of the quality of life measurements. American Medical Association Journal 272, 24-31.

Kind, P. \& Dolan, P. (1995) The effect on past and present illness experience on the valuations of health states. Medical Care 33, 255-263.

Leplege, A. \& Sonia, H. (1997) The problem of quality of life in medicine. American Medical Association Journal 278, 47-50.

Maclachlan, M. (1996) Identifying problems in community health promotion: an illustration of the nominal group technique in AIDS education. Journal of Royal Society of Health 116, 43-48.

Makundi, E., Haram L., Norheim, O. \& Setel, P. (2004) Community social valuation: lay peoples' understanding of physical disability in two communities in Tanzania. Tanzania Health Research Bulletin 6, 18-24.

McMurray, A. (1994) Three decision-making aids: brainstorming, nominal group technique and Delphi technique. Journal of Nursing Staff Development 10, 62-65.

Miller, G.A. (1956) The magical number seven plus or minus two: some limits on our capacity to process information. Psychology Review 63, 81-97.

Murray, C. \& Lopez, A. (1996) Global Burden of Disease. World Health Organization. Geneva.

Murray, C. \& Lopez, A. (1997) Global mortality, disability and the contribution of risk factors: Global burden of disease study. The Lancet 349, 436-442.

Nord, E. (1992) Methods for quality adjustment of life years. Social Science and Medicine 34, 559-569. 
Nord, E. (1995) The person-trade-off approach to valuing health care programs. Medical Decision Making 15, 201-208.

Nord, E. (1999) Cost-value Analysis in Health Care: Making Sense Out of QALYs. Cambridge University Press.

Redman, S., Carricks, S., Cockburn, J. \& Hirist, S. (1997) Consulting about priorities for the NHMRC National Breast Cancer Centre: how good is the nominal group technique. Australia and New Zealand Journal of Public Health 21, 250-256.

Sayers, B.M. \& Fliedner, T.M. (1997) The critique of DALYs: a counter reply. Bulletin of World Health Organization 75, 383-384.

TDHS (1992/1993) Tanzania Demographic and Health Survey. Planning Commission, Dar es Salaam, Tanzania.
Torrance, G.W. (1986) Measurement of health state utilities for economic appraisal. Journal of Health Economics 5,1-30.

Torrance, G.W., Boyle, M.H. \& Horwood, S.P. (1982) Application of multi-attributive utility theory to measure social preferences for health states. Operational Research 30, 1043-1069.

Ustun, B.T., Rehm, J., Chtterji, S., Saxena, S., Trotter, R., Room, R. \& Bickenbarch, J. (1999) Multiple-informant ranking of the disabling effects of different health conditions in 14 countries. The Lancet 354, 111-115.

Walton, R.E. (1985) From control to commitment in work place. Harvard Business Review: March-April, 77-84.

WHO (1998) Report on the Informal Consultation on DALYs and Reproductive Health. World Health Organization, Geneva. 